\title{
A Refined X-Band Microwave Microcalorimeter
}

\author{
Glenn F. Engen
}

\author{
(May 1, 1959)
}

\begin{abstract}
The microcalorimetric method for evaluation of the efficiency and substitution error of a bolometer mount proposed by Macpherson and Kerns has been the object of further study and refinement at the Boulder Laboratories of the National Bureau of Standards, and an improved instrument based on this technique has been recently placed in operation.

The new microcalorimeter design features are: (1) Greatly improved ambient temperature control, permitting higher sensitivity and resolution; (2) improved d-c instrumentation; (3) improved mechanical construction giving better repeatability; (4) relocation of the thermopile so that it no longer is attached directly to the bolometer mount, thus providing flexibility in the choice of termination; and (5) a more comprehensive treatment of the calorimetric substitution or equivalence error. These features permit the determination of the effective efficiency of a bolometer mount to an accuracy of better than 0.2 percent.
\end{abstract}

\section{Introduction}

The bolometric technique, by means of which the heating effect of an unknown amount of rf or microwave power is compared with that of a measured amount of $d-c$ or audiofrequency power through a temperature-sensitive resistive element, is a wellknown and extensively employed method in the low-level measurement of microwave power.

In a commonly used arrangement, the bolometer element forms one arm of a Wheatstone bridge, the parameters of which are chosen in such a manner that the bridge is balanced when the bolometer is at its nominal operating resistance. The bolometer is then provided with the required bias power to bring its resistance to this operating value, and the reduction in bias power required to maintain the bridge balance following the application of a microwave signal, is taken as a measure of the microwave power. The measurement of this bolometric or substituted d-c bias power has been the object of refinement until the measurement can be carried out to an accuracy of 0.1 percent [1]. ${ }^{1}$

The use of the value thus obtained as a measure of the microwave power involves, however, two well-recognized sources of systematic error. First, the microwave and $\mathrm{d}-\mathrm{c}$ bias currents take different distributions within the bolometer element, which means that the functional dependence of the bolometer resistance will, in general, be different for the two sources of power, giving rise to a dc-rf substitution error. Second, the bolometric method at best measures only the microwave power dissipated within the bolometer element, whereas one is usually interested in the power dissipated in the entire terminating waveguide structure or bolometer mount. The ratio of these quantities is, by definition, the bolometer mount efficiency. In addition, if the mount is not properly tuned there may be a net reflection of power at its input terminals, but the methods of dealing with this phase of the problem are well-recognized [2] and will not be considered explicitly in this paper.

${ }_{1}^{1}$ Figures in brackets indicate the literature references at the end of this paper.
Macpherson and Kerns [3] gave the details of a calorimetric method for the determination of the combined effect of these two sources of error. This technique has been the object of further study and refinement at the Boulder Laboratories, and an improved instrument based on this method was recently placed in operation. A number of calorimetric-type power meters have also been developed $[4,5,6]$ in other laboratories which utilize the same general techniques employed by Macpherson and Kerns, but the further development at the NBS has retained the original objective of obtaining a correction factor for the bolometrically-determined power.

\section{General Description}

The basic theory of operation has been presented in detail in the cited references and need not be repeated here. In brief, however, the instrumentation is such as to permit a calorimetric determination of the total power input to a bolometer mount while a simultaneous bolometric determination is made. The difference between the two measurements is ascribed to the mount efficiency and substitution error. The method, in its present state at least, permits only a determination of the combined effect of these phenomena, referred to as "effective efficiency."

The problem of making a calorimetric determination of power (or energy) may be subdivided as follows: (1) Selection of a calorimetric body or object in which to dissipate the power to be measured, (2) choice of a suitable technique to measure the temperature rise of this body or object, and (3) determination of the proportionality factor or functional dependence of the thermometer response upon the power input. In the microcalorimeter technique, the bolometer mount serves as the calorimetric body or object in which the power is dissipated and whose temperature rise is measured by means of a suitable thermopile, while calibration is effected by observing the thermopile response to a measured amount of d-c power dissipated in the bolometer element. 
The thermopile consists of 34 constantanchromel-P junctions with an approximate senstivity of $2,000 \mu \mathrm{v} /{ }^{\circ} \mathrm{C}$. The instrument is designed to yield a nominal output at equilibrium of $100 \mu \mathrm{V}$ corresponding to a temperature rise of $0.05^{\circ} \mathrm{C}$. Since this potential may be measured by the associated instrumentation to an accuracy of a few parts in $10^{4}$, a temperature background which is stable to a few millionths of a degree is indicated for the application.

In order to achieve this background, the twin Joule arrangement is employed as shown in figures 1 and 2, along with a temperature-regulated water bath. The advantage of this method stems from the fact that if symmetry exists with respect to a plane normal to the plane of the illustration as shown, and if no thermal gradients exist within the thermal shields, then the actual temperature of these shields may fluctuate without producing a temperature difference between the bolometer mounts. Thus, by using a triple thermal shield (the innermost one of which is a heavy copper casting), a symmetrical waveguide input system, and suitable thermal shunts, a degree of symmetry is achieved such that the dependence of the temperature difference between the bolometer mounts upon the thermal head existing between the inner thermal shield and the external water bath, is only a factor of approximately one part in $10^{4}$. Since a bath regulation of $\pm 0.005^{\circ} \mathrm{C}$ may be obtained with little difficulty, adequate isolation from the bath fluctuations is thus achieved. There still exist in the current model, however, temperature gradients whose origin is yet undetermined such that the thermal background is stable to only 5 to 10 millionths of a degree, but this is satisfactory for the application.

In order to function in the required manner, thermal isolation is required between the bolometer mounts and the remainder of the system. (Isolation is also required between the thermal shields.) This rules out the possibility of using conventional waveguide to convey microwave energy to the bolometer mounts, since this would also readily conduct away the heat generated in the mount. In the earlier instrument, this isolation took the form of choke-flange-air-gap combination, but such an

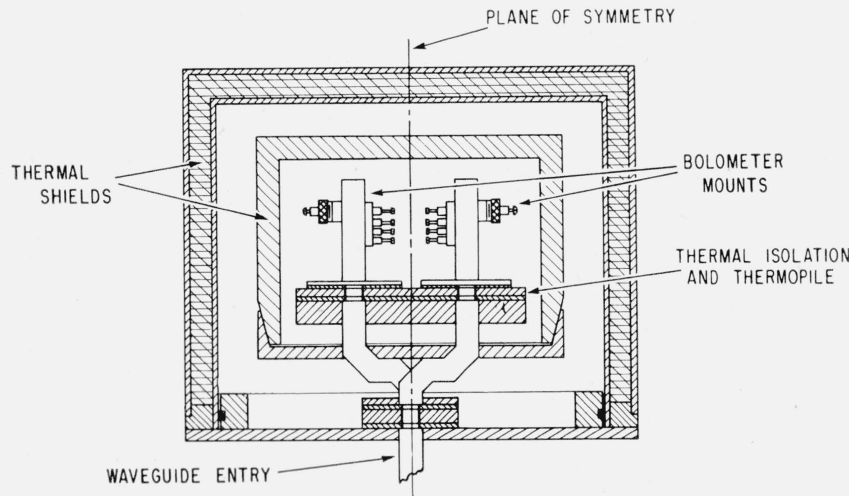

Figure 1. Cross section of microcalorimeter.

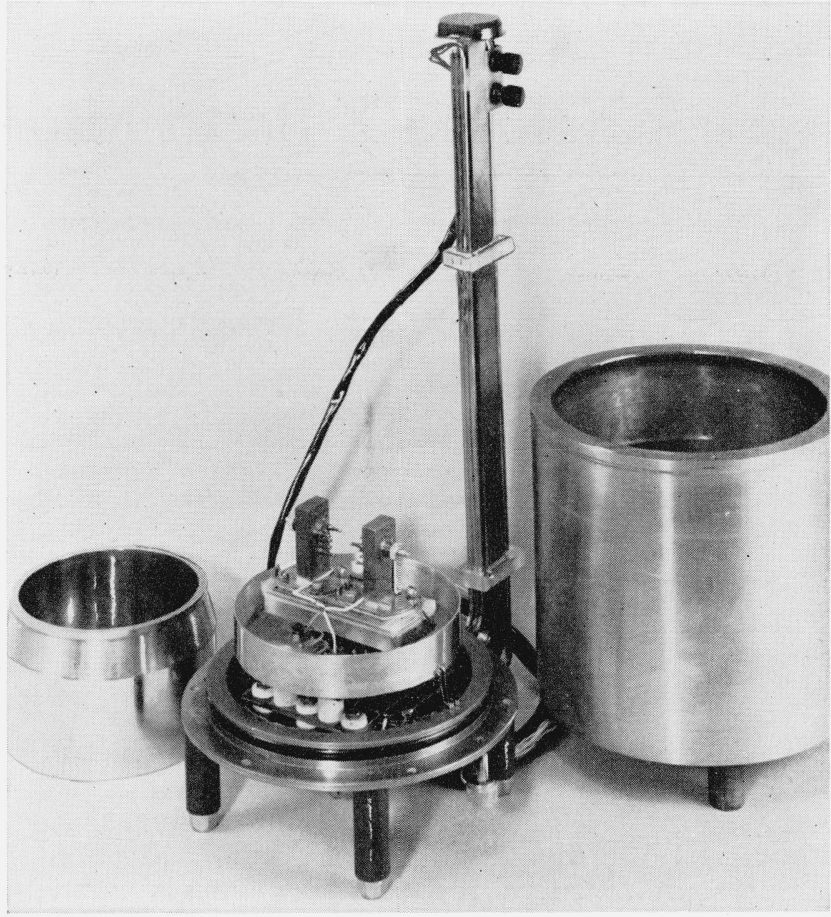

Figure 2. Microcalorimeter and thermal shields.

arrangement at best is frequency sensitive, and at worst, may introduce systematic errors whose evaluation is quite difficult. In the present model, this isolation is provided by a short section of waveguide, the body or sides of which are fabricated from 0.001-in. copper shim with the addition of suitable plastic to provide the required mechanical rigidity.

A significant increase in the flexibility and operating convenience of the instrument has been realized through relocation of the thermopile. Instead of being attached directly to the bolometer mounts as in the former model, the thermopile is built into the thermal isolation sections in such a way that it senses the temperature difference between the flanges which mate with the bolometer mount flanges as shown in figures 3 and 4 . The obvious advantage of this arrangement is that removal of the four screws which hold the bolometer mount in place in the colorimeter also serves to remove the mount from the thermopile assembly, thus permitting flexibility in the choice of termination and enabling the ready duplication or multiplication of the number of bolometer mounts which may be so calibrated.

There are a number of design criteria which the bolometer mounts employed in the calorimeter must satisfy. First, a high degree of stability in those parameters which determine the mount efficiency is obviously desirable, while the ability to tune the mount for an impedance match (no reflection) at the operating frequency is also desirable if comparison with other mounts is anticipated. The choice of the tuning elements which may be employed in achieving this impedance match is severely limited by the necessity of keeping the mass (heat capacity) of the entire mount within prescribed limits. In 


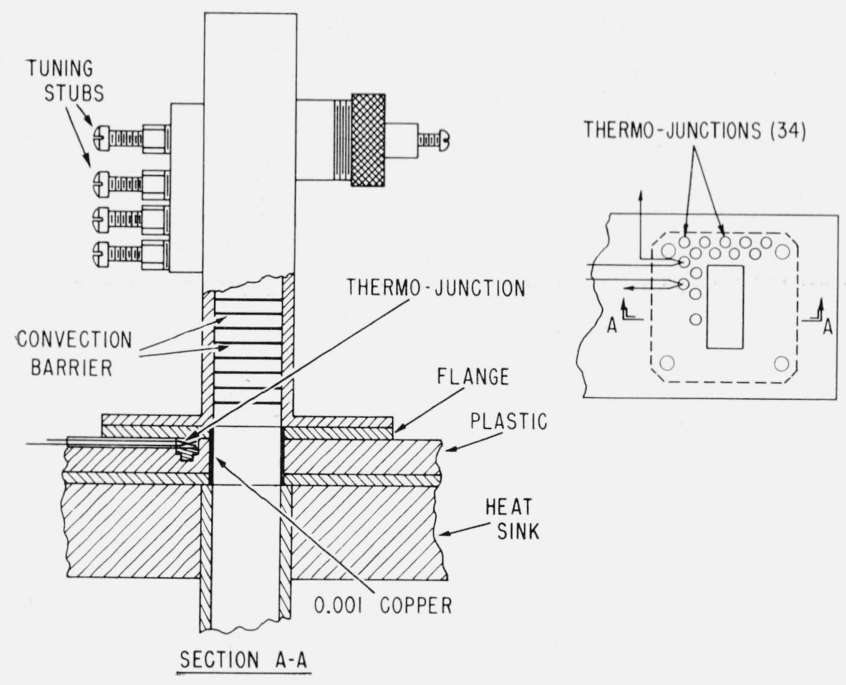

Figure 3. Detail of thermopile construction.

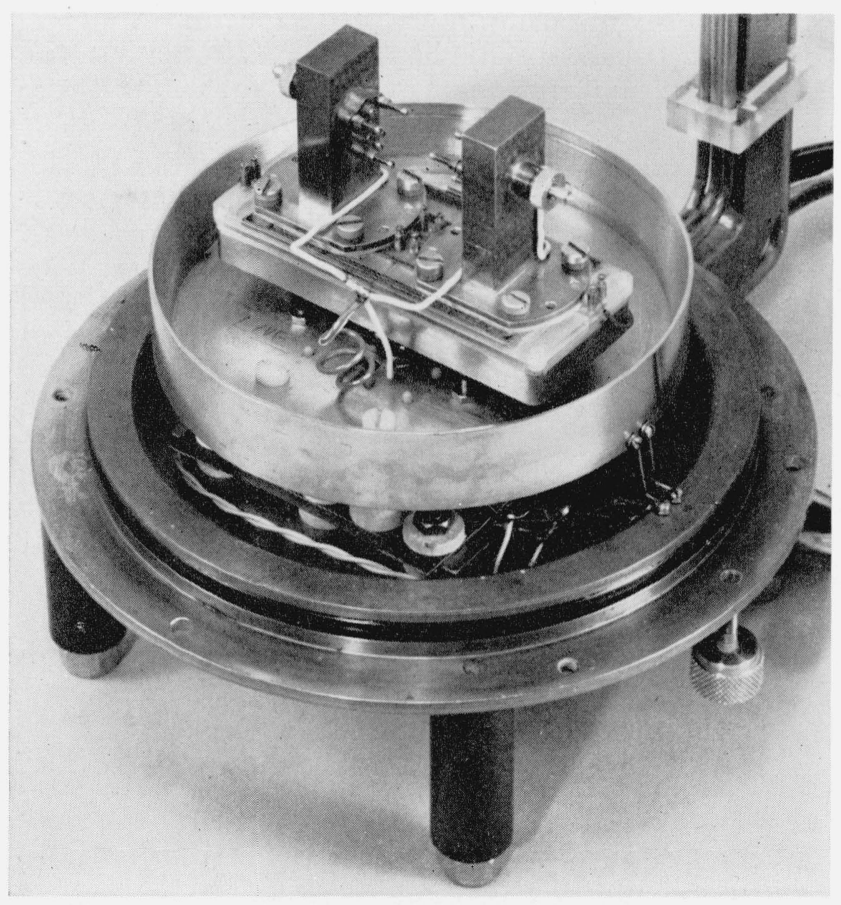

Figure 4. Closeup of microcalorimeter interior.

addition, while comparatively little is yet known of the distribution of losses within a bolometer mount, many of the commonly employed tuning elements would appear to be potential sources of instability in the mount efficiency.

Another requirement on the mount design is that the calorimetric equivalence error (to be discussed later) be held to a small value; that is, the thermopile response should be essentially independent of the distribution of the heat sources within the mount. This will be true if the mount is of uniform temperature overall, which suggests the use of thick walls and keeping the length short. The design should also provide a high value for the efficiency as this will also reduce the error from calorimetric nonequivalence. Some of these problems will be discussed in greater detail in later sections, but it will be recognized from the present discussion that the design must effect a compromise among a variety of conflicting requirements.

\section{Operational Procedure}

The operational procedure associated with the instrument is comparatively simple and straightforward. Using the terminology introduced by Macpherson and Kerns [3] an effective efficiency, $\eta_{e}$, may be defined,

$$
\eta_{e}=\frac{\text { Substituted or retracted bias power }}{\text { Total microwave power dissipated within }} \text {. }
$$

In practice the bolometer is operated in conjunction with a selfbalancing d-c bridge [1]. The d-c bias power is first applied and the thermopile response allowed to effectively reach an equilibrium value (which takes $45 \mathrm{~min}$ ). Microwave power is then applied, d-c power is withdrawn as required to maintain bridge balance, and the thermopile response again permitted to reach equilibrium, at which time the microwave source is removed and the thermopile response to d-c power only once more observed. The procedure thus consists of energizing the bolometer element from the $\mathrm{d}-\mathrm{c}$ bridge and observing the thermopile response and bridge current, without and with microwave energy present in 45-min intervals. Ideally, only one cycle of microwave power off and on would be required, but in practice averaging the results of two "off" intervals occurring before and after an "on" interval permits further suppression of the thermal background drift. Typical thermopile response curves are shown in figures 5 and 6.

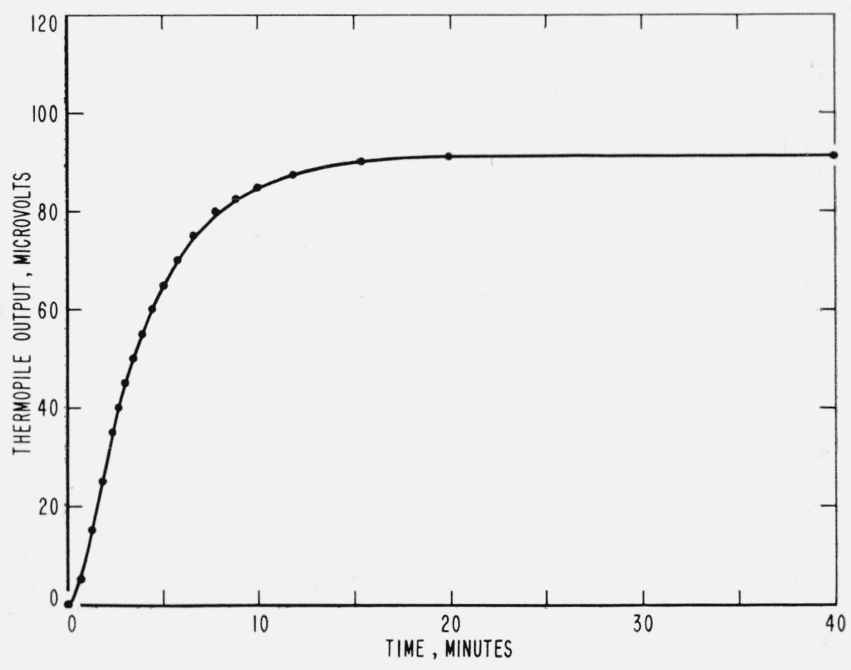

Figure 5. Typical thermopile response. 


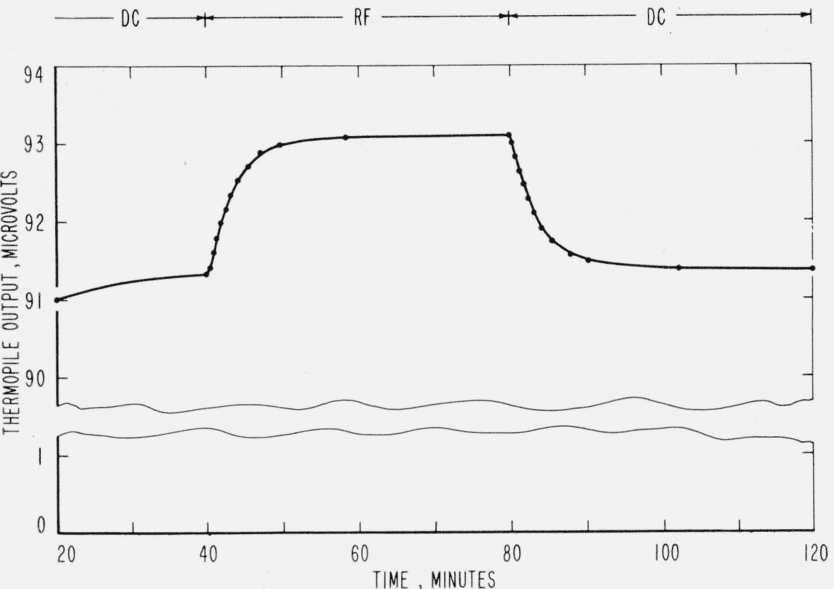

Figure 6.-Response curve for determination of effective efficiency.

Let $i_{1}$ and $i_{2}$ represent the bolometer bridge currents without and with microwave energy present in the bolometer and, respectively, $e_{1}$ and $e_{2}$ the corresponding thermopile responses. Then for the equal arm bridge in common use, the bolometric power is given by the usual formula,

$$
P_{b}=\frac{r_{0}}{4}\left(i_{1}^{2}-i_{2}^{2}\right)
$$

where $r_{0}$ is the resistance of the bolometer element and bridge arms.

The total power input to the bolometer mount (microwave and d-c) during the microwave "on" interval ideally will be proportional to the thermopile response, $e_{2}$, the proportionality factor being $r_{0} i_{1}^{2} / 4 e_{1}$. The microwave power is the difference between this total input and the d-c component, thus

$$
P_{r f}=\frac{r_{0}}{4}\left(\frac{e_{2}}{e_{1}} i_{1}^{2}-i_{2}^{2}\right)
$$

and

$$
\eta_{e}^{\prime}=\frac{1-\left(i_{2} / i_{1}\right)^{2}}{e_{2} / e_{1}-\left(i_{2} / i_{1}\right)^{2}}
$$

which gives the effective efficiency $\left(\eta_{e}^{\prime}\right)$ in terms of the thermopile response and bolometer-bridge currents. The prime is used to indicate that the equation is based on the assumptions that the thermopile response is linear and independent of the distribution of the power dissipation within the mount. The validity of these assumptions will be examined below.

\section{Error Analysis}

Equation (2) for $\eta_{e}^{\prime}$ has been obtained under certain simplifying assumptions; a more general approach to the problem will now be presented. Within the limits of experimental error (to be discussed below), the thermopile response and cooling mechanisms are both linear, and the thermopile response $e_{2}$ is of the form:

$$
e_{2}=k\left(P_{2_{d c}}+g P_{r f}\right)
$$

where $k$ and $g$ are constants, and $P_{2_{d c}}$ and $P_{r f}$ are the d-c power dissipated in the bolometer element and the total microwave power dissipated in the bolometer mount, respectively. Equation (3) expresses the fact that a given amount of microwave power will, in general, produce a different thermopile response than an equal amount of $d$-c power through the factor $g$.

The constant $k$ may be determined in the manner already discussed so that $k=e_{1} / P_{1_{d c}}$. Substituting this result and solving eq (3) for $P_{r f}$ gives,

$$
\begin{gathered}
P_{r f}=\frac{1}{g}\left(\frac{e_{2}}{e_{1}} P_{1_{d c}}-P_{2_{d c}}\right) \\
\eta_{e}=g \frac{P_{1_{d c}}-P_{2_{d c}}}{\frac{e_{2}}{e_{1}} P_{1_{d c}}-P_{2_{d c}}}=g \frac{1-\left(\dot{i}_{2} / i_{1}\right)^{2}}{e_{2} / e_{1}-\left(i_{2} / i_{1}\right)^{2}}
\end{gathered}
$$

The more complete analysis thus gives a factor $g$ by which the right-hand side of $\mathrm{eq}(2)$ is to be multiplied in determining $\eta_{e}$. The error in determining $\eta_{e}$ thus consists of the uncertainty in the value of $g$, and the experimental error in determining the value of this second factor, $\frac{1-\left(i_{2} / i_{1}\right)^{2}}{e_{2} / e_{1}-\left(i_{2} / i_{1}\right)^{2}}$. These will be referred to as the calorimetric equivalence and instrumentation errors, respectively.

The instrumentation error may be evaluated with little difficulty. If the second factor is differentiated with respect to $i_{1}$ and $i_{2}$, it is found that for typical values of the ratio $e_{2} / e_{1}$; i.e., $1<e_{2} / e_{1}<1.05$, the error in $\eta_{e}$ is only 5 percent or less of the error in $i_{1}$ or $i_{2}$. In practice, $i_{1}$ and $i_{2}$ are measured by a precision potentiometer-standard resistor combination to an accuracy of about 0.03 percent; thus, the total error due to the uncertainty in $i_{1}$ or $i_{2}$ is only of the order of 0.01 percent and may be neglected.

The error due to the uncertainty in $e_{2} / e_{1}$ may be found in a similar manner, and if the ratio of $i_{2}$ to $i_{1}$ is $1 / 2$ or less, the error in $\eta_{e}$ is in the range 1.0 to 1.3 times the error in $e_{2} / e_{1}$.

In order for eq (3) to be valid, the thermopile response must be a linear function of the $d-c$ and microwave powers. The error in the ratio $e_{2} / e_{1}$ may conveniently be defined to include, in addition to the instrumentation error in measuring the thermopile output, the error due to nonideal performance of the calorimeter caused by such things as nonlinear thermopile response and cooling, thermal-background drift, etc., which might affect the validity of eq (3). The error in measuring the ratio $e_{2} / e_{1}$ was determined in the following manner. The measurement procedure outlined in the foregoing section was followed except that instead of applying microwave power at the end of the first 45-min interval, the d-c power applied to the bolometer was increased by a nominal 10 percent. In this way the actual operating conditions were simulated, and the measured value of this second d-c power was compared with that predicted on the basis of a linear response from the observation of $e_{1}, e_{2}$ and the first value of d-c power. 
In ten measurements of this type, the maximum error observed was 0.04 percent, with an average error of 0.013 percent. A figure of 0.1 percent, corresponding to an error in $e_{2} / e_{1}$ of 0.07 percent, has been arbitrarily assigned as a conservative limit to the error from this source, in which the probable error is perhaps 0.02 percent or less.

With regard to the equivalence error, $g$ is a number which expresses the relative effectiveness of microwave power as compared with d-c power in producing a thermopile response, that is, for a given thermopile output, $g=P_{d c} / P_{r f}$. Ideally, $g=1$ but in practice differs from this value due to the following phenomena:

1. The $d-c$ and microwave powers take different distributions within the bolometer element yielding different thermopile responses.

2. Some of the microwave energy will be dissipated in places other than the bolometer element and the thermopile response will again differ from that produced by an equal amount of $d-c$ power in the bolometer.

3. The thermopile will respond to the dissipation of microwave energy in the waveguide lead-in which does not comprise part of the bolometer mount.

It is just these first and second phenomena, of course, which are responsible for the dc-rf substitution error and mount inefficiency.

With reference to the first of these, the temperature-sensing device in the calorimeter is relatively far removed from the main heat source and it might be estimated conservatively that the error in the calorimetric method due to this phenomena would be at least an order of magnitude or more below the bolometric substitution error which according to the information available [7], does not exceed a few percent. $^{2}$

In order to check the validity of this assumption experimentally, the $\mathrm{d}$-c calibration factor, $k$, was determined for several different bolometer elements (barretter and thermistor). The initial results of this experiment gave values which differed from one another by as much as 2 percent with a repeatability of 0.1 percent. This anomaly at first appeared to challenge the validity of the above assumption, but further investigation showed that the insertion of a 0.05 in. copper plate at the bolometer-mount input flange (thus blocking the waveguide input), would eliminate this difference. This was interpreted to mean that air convection was playing a significant role in cooling the bolometer capsule (not to be confused with the cooling of the bolometer element within the capsule), and the variations in the value for $k$ were ascribed to the variable role, occasioned by the differences in the capsules associated with the bolometers, played by air convection in conveying this heat from the capsule to the external heat sink via the interior of the waveguide.

By inverting the bolometer mounts such that the closed end was up, it had been hoped that the need

${ }^{2}$ Recent advances in the impedance method of measuring bolometer mount efficiency have demonstrated that the substitution error at frequencies near 10 $\mathrm{kMc}$ is probably no greater than a few tenths of a percent. for a convection shield or barrier could be eliminated, but these results clearly demonstrated the need for such a shield. In order to provide a convection barrier, in which the microwave dissipation would be negligible, a series of eight 0.002 in. Teflon membranes spaced at 0.05 in. intervals and transverse to the waveguide axis was arranged at the waveguide input. This effectively eliminated the convection problem and gave repeated determinations of $k$ in a refined bolometer mount which agreed to 0.05 percent, which is about the limit of experimental error for this particular measurement. Since the power distributions in the barretter and thermistor are markedly different, it may be safely assumed that the small difference in distribution of the $d-c$ and microwave powers which occurs in practice when the same element is employed, will have a negligible effect.

With regard to the degree of equivalence realized in the thermopile output for power dissipated in the bolometer mount walls as compared with an equal amount in the bolometer element, the problem, once again, is primarily that of attempting to prevent the escape of heat from the mount via air convection. The effectiveness of the convection barrier described in the previous paragraph was demonstrated by temporarily inserting two other heat sources within the mount such that they were in good thermal contact with the side walls. The first of these was located just inside the mount before the teflon barrier, the second on the other side of the barrier, close to the bolometer element as shown in figure 7 . The thermopile response for equal amounts of power in the two positions was then compared with that for the bolometer element, and it was found that the heat sources at positions 1 and 2 were approximately 1.5 and 0.5 percent more effective respectively. A conservative figure for the increased effectiveness of the power dissipated in the walls is thus 1 percent with an error of \pm 1 percent. It should be noted that this figure applies only to that fraction of the power which is so dissipated. In practice a lower limit of 97 percent may be taken for the efficiency of the mounts designed for use in this calorimeter, which leaves only 3 percent of the energy to be dissipated other than in the bolometer element.

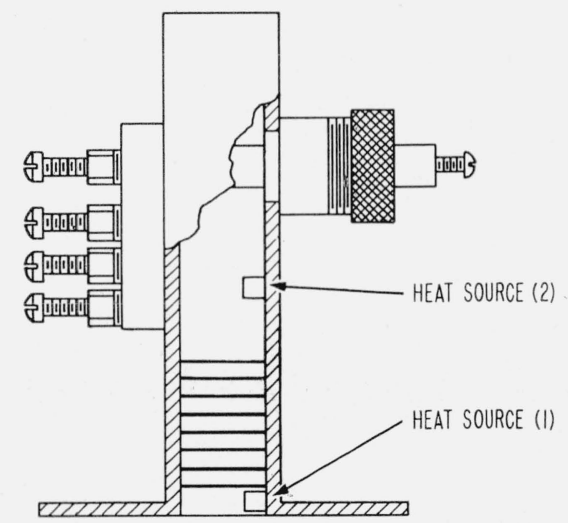

Figure 7. Bolometer mount with auxiliary heat sources. 
Finally, as noted, the thermopile will respond to the dissipation of microwave energy in the thermal isolation section which is not part of the bolometer mount. If an electrical conductivity of 50 percent of the $d-c$ value is assumed for this $1 / 4$ in. length of copper waveguide, the theoretical value of power dissipation will be approximately 0.05 percent, of which the thermopile will probably measure somewhat more than half. A value of 0.04 percent \pm 0.04 percent thus represents a conservative estimate for this contribution.

The results of this section are summarized in table 1.

TABLE 1

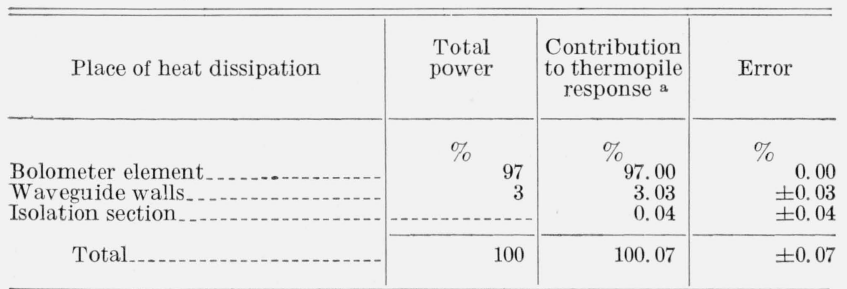

a Compared with an equal amount of d-c power dissipated in the bolometer element.

Rounding off these results gives for $g$ a value 1.001 with a possible error of \pm 0.1 percent; and this, combined with the instrumentation error of \pm 0.1 percent, gives for the total error \pm 0.2 percent.

\section{Bolometer Mount Design and Experi- mental Results}

As may be inferred from the foregoing description, the design of the bolometer mounts employed in the microcalorimeter has undergone a series of transitions as required to cope with the various engineering problems encountered in the evaluation of the device. While no claim is made as to the extent of their applicability, certain features of the construction are believed to be of enough interest to merit further description.

The general features of the construction are shown in figures 4 and 7 . The mount is machined in two halves from a solid piece of tellurium copper, and the two halves hard-soldered together along the center of the wider side. The brass flange is then attached by soft solder. Except for these two applications, the use of solder has been completely avoided in the construction. The incentive for this method of fabrication was provided by the observation of efficiencies of the order of 85 percent for mounts in which the back plate was fastened with soft solder, while the use of hard solder increased these values to $95-98$ percent.

The design employs five tuning stubs as shown, three in front of the bolometer and two at the side. These side stubs provide additional susceptance at the plane of the bolometer element, and thus, over a limited range, serve the same function as a moveable back plate, while the other three stubs serve the usual purpose.

In the five mounts which have been built to this general pattern, effective efficiencies in the vicinity of 98 to 99 percent have been realized. The results of additional measurements to determine the place of this residual dissipation seem to imply that most of it occurs in the plastic capsule surrounding the element and/or in the microwave bypass mechanism, at the d-c lead-in to the bolometer. While additional research will be required to completely resolve these questions, it appears likely, on the basis of present information, that effective efficiences of 99.5 percent and perhaps higher may be realized by proper design and fabrication techniques.

Finally, table 2 shows the results of intercomparisons with commercially available mounts.

TABLE 2

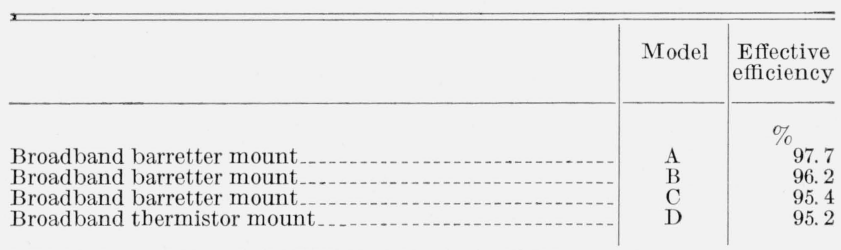

The author acknowledges the assistance of Morris E. Harvey, to whose care in making the experimental observations a large amount of credit for the success of this project is due, and extends his thanks to R. W. Beatty and D. M. Kerns for their helpful suggestions in the preparation of the manuscript.

\section{References}

[1] G. F. Engen, A self-balancing d-c bridge for accurate bolometric power measurements, J. Research NBS 59, 101 (1957) RP2776.

[2] R. W. Beatty and A. C. Macpherson, Mismatch errors in microwave power measurements, Proc. IRE 41, 1112 (1953).

[3] A. C. Macpherson and D. M. Kerns, A microwave microcalorimeter, Rev. Sci. Instr. 26, 27 (1955).

[4] M. Sucher and H. J. Carlin, Broad-band calorimeters for the measurement of low and medium level microwave power. I. Analysis and design, IRE Trans. on Microw. Theory Tech. 6, 188 (1958).

[5] A. V. James and L. O. Sweet, Broad-band calorimeters for the measurement of low and medium level microwave power. II. Construction and performance, IRE Trans. on Microw. Theory Tech. 6, 195 (1958).

[6] W. M. Sharpless, A calorimeter for power measurements at millimeter wavelengths, IRE Trans. on Microw. Theory Tech. $\mathbf{2}, 45$ (1954).

[7] H. J. Carlin and M. Sucher, Accuracy of bolometric power measurements, Proc. IRE 40, 1042 (1952).

Boulder, Colo.

(Paper 63C1-9) 